\title{
TREATMENT OF CHEMICAL BURNS OF THE EYE WITH CORVASYMTON*
}

BY

\author{
J. W. WAGENAAR \\ Alkmaar, Netherland:
}

THE results of the experimental investigation carried out in the laboratory of the Netherlands Ophthalmic Hospital in Utrecht to test my first clinical findings on the favourable action of "Corvasymton " + in a chemical burn of the eye have already been recorded (Fischer, Hodgson, and Wagenaar, 1950). In view of the favourable result I decided to use this drug once again when a patient with a chemical burn should come to me for treatment. Shortly afterwards I had the opportunity of administering Corvasymton in an extremely severe case of calcium oxide corrosion, which I believed at first to stand no chance of recovery.

\section{Case Report}

A 10-year-old boy, whose face had been struck by a piece of quicklime while playing a little under an hour before, was brought to me on October 10, 1949. Plentiful washings with water had already been given at the family practitioner's surgery.

The condition appeared uncommonly severe. Numerous particles of lime were retained between the eyelids and deep in the fornices as the result of a violent blepharospasm. There was a marked bilateral chemosis, the conjunctivae showed a brown-grey discoloration and the vessels were black in so far as they were visible, so that they had presumably become thrombosed.

Right Eye: The entire cornea was a dull porcelain-white, so that nothing could be seen of the iris. Vision was naturally limited to the perception of light.

Left Eye: A small part of the upper cornea was still slightly transparent, so that a small portion of the iris was visible (Fig. 1). Visual acuity was limited to the perception of coarse shapes at a few metres' distance and could be roughly estimated at $2-3 / 300$.

As a preliminary treatment, as many particles of lime as possible were removed with moist cotton-wool carriers, and I also instilled Corvasymton. At the hospital I was able to carry out a complete cleansing under ether anaesthesia, which showed that various particles of lime had burnt deep into the conjunctiva. Thereupon the apparently entirely necrotic portions of the conjunctivae along the limbus corneae were loosened-up in the sector from 4 to 8 o'clock and extensively undermined in a peripheral direction (Fig. 2). This procedure did not give rise to any haemorrhages, which indicates that the conjunctival vessels had been completely occluded. The only subsequent treatment consisted of instillations of Corvasymton, every half hour in the day-time and every hour at night.

Within 24 hours the conjunctivae had largely regained their pink colour and the chemosis had nearly disappeared, and the upper part of the right iris also became visible. During the following days the porcelain-white cloudiness gradually disappeared,

* Received for publication November 29, 1951.

+ An oxyphenyl-methylamino-ethanol tartrate solution of Philips-Roxane Ltd. 


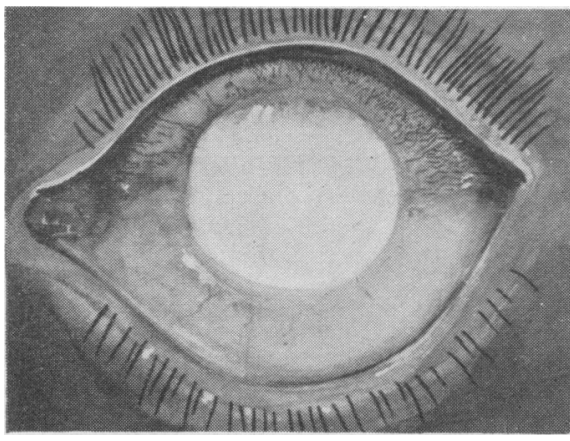

FiG. 1.-Sketch of left eye shortly after injury.

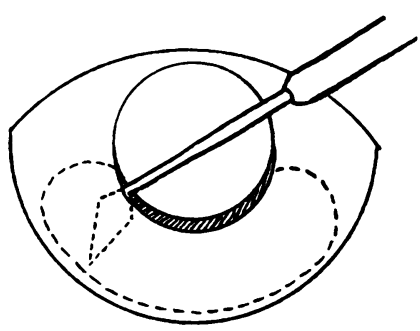

FIG. 2.-Diagram of undermining "necrotic" conjunctiva with a narrow keratome.

to be replaced by a diffuse cloudiness of both corneae, through which, however, the whole iris could be perceived. Since after one week the corneal epithelium failed to show any signs of recovery, I had oil of vitamin A and Corvasymton administered alternately during the second week, and then oil of vitamin A alone for a few days longer. Though the corneal epithelium was now recovering fairly rapidly, the corneal cloudiness increased considerably, and the visual acuity, which had increased to about $1 / 60$, once more diminished. As soon as Corvasymton was instilled again, both corneae cleared up.

Since a perforation of the very weak-looking cornea appeared to be imminent, I did not at first venture to give a mydriatic. From November 1 the patient received 0.5 per cent. atropine once a day, by means of which a satisfactory mydriasis could be maintained.

On November 15 the visual acuity was $1 / 60$ in the" right eye and 5/50 in the left eye with error ; stenopaeically it was even $5 / 20$ with error (Figs 3 and 4). A highly
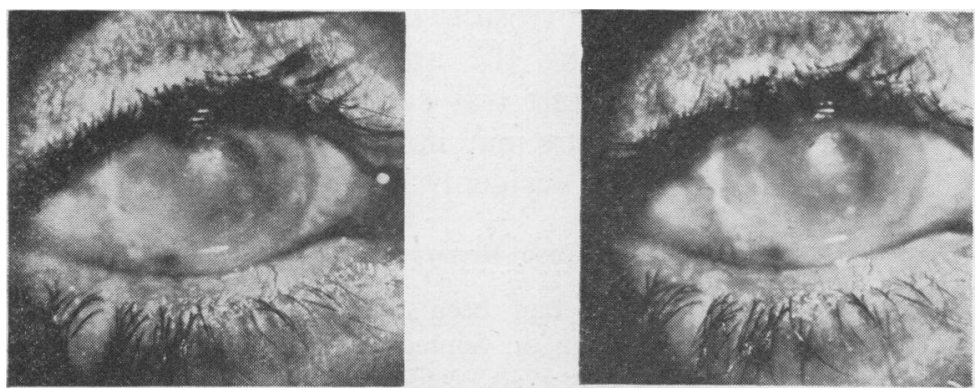

Fig. 3.-Right eye 5 weeks after injury.
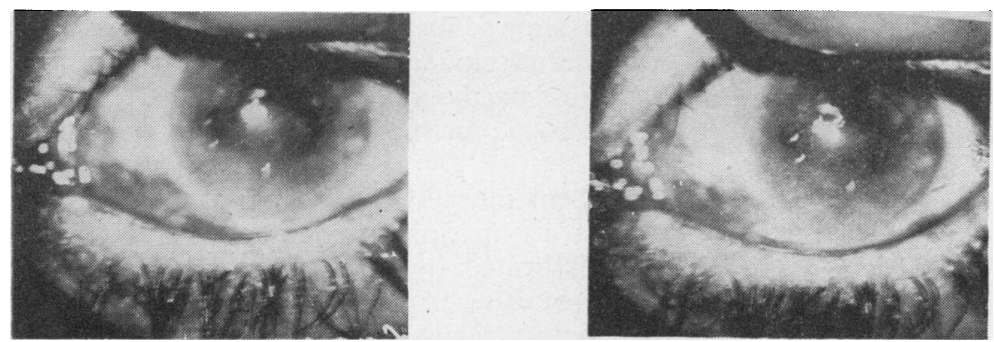

FIG. 4.-Left eye 5 weeks after injury. 
irregular astigmatism rendered any further cor̂rection impossible. At my request my colleague Mrs. H. Fischer von Bünau was so kind as to examine the patient with contact lenses, with the amazing result that the visual acuity could be improved in the right eye to $5 / 6.6$ with error, and in the left eye to $5 / 10$ with error. This meant that this boy would at any rate be able to return to normal life. In the beginning of January, 1950, a common correction with glasses of his compound hypermetropic astigmatism resulted in the patient's visual acuity being 5/10 with error when both eyes were used, and he is now once more able to attend normal instruction, aided only by a pair of spectacles.

Denig (1912) was the first to suggest a replacement of the necrotic conjunctivae in corrosions by oral mucous membrane, which procedure was assumed not only to counteract the formation of symblepharon, but also to assist the clarification of the clouded cornea. Thies (1928) once more drew attention to the favourable results obtained by this operation, which has since become known as the Denig-Thies oral mucosa graft.

Passow (1939) called attention to the fact that the mere loosening-up of the corroded conjunctiva produces a result similar to that of the complete Denig-Thies operation, which treatment naturally also entails the liberation of the involved conjunctiva of the limbus corneae. This is believed to be explained by the fact that a deposit of the agent accumulates under the conjunctiva, thus impairing the conjunctival, scleral, and corneal nutrition. Presumably for the same reason, Pichler (1910), Middleton (1935), and Oaks (1945) recommended a paracentesis of the cornea. Oaks reports that turbid aqueous humour escaped during this procedure, which was done shortly after the corrosion; this also testifies to the rapid action, which may give rise to the formation of deposits.

In two cases of conjunctival corrosion I was able to obtain an equally favourable result by undermining the apparently necrotic parts of the conjunctiva, so that a mucosal graft was superfluous. The following case shows how such a simple procedure may have a favourable effect even when carried out several days after the accident.

\section{Case Report}

A 38-year-old man, whose left eye had been splashed by a drop of strong $\mathrm{NaOH}$ solution on September 14, was seen by me on Septem-

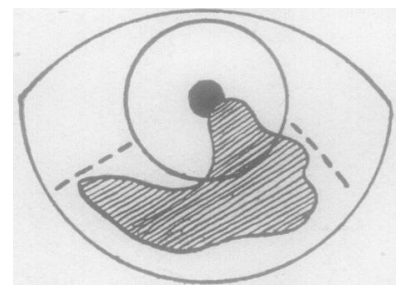

Fig. 5.-Epithelial defect of cornea and "necrotic" area of conjunctiva 6 days after. injury; dotted lines indicate starting points of undermining with keratome. 
colour and the patient was fit to be discharged from hospital on October 9. On January 22, 1949 , the visual acuity could be corrected to $5 / 10$ with $S-0.5$; a slight pseudopterygium which has shown no subsequent increase developed, as well as a slight symblepharon which did not call for correction.

In the first-mentioned case of severe calcium oxide corrosion, in which the conjunctivae along the limbus corneae were loosened-up in both eyes in so far as they seemed necrotic, the right eye has developed an insignificant symblepharon, which does not require surgical treatment since it causes no inconvenience. Thus this case once more showed how a surprising recovery of the conjunctiva may be obtained by the simple procedure of loosening it up in so far as it appears to be injured; moreover this enables the Corvasymton to exert a direct subconjunctival action, which is an undoubted advantage.

In my opinion one should not speak of a " necrotic" conjunctiva, even though all the vessels are found to be thrombosed or otherwise occluded. When the tissue has an opportunity of recovering within a relatively short time after the accident, it presumably stands the same chance as the graft of oral mucosa, which has also to be revascularized. In his excellent study on alkali burns of the cornea, Hughes (1946) also voices his doubt as to the value of grafts with oral mucosa:

The early excision of necrotic conjunctival and episcleral tissue and its replacement with oral mucous membrane (Denig-graft) or conjunctiva has been employed by many clinicians for the treatment of severe alkali burns. Analysis of these reported cases does not reveal any striking benefit attributable to such a procedure.

Heinsius (1949) recalls Löhlein's opinion that one should only consider an excision of necrotic conjunctival tissues without transplantations of mucous membrane if one wishes to prevent a vascularization of the cornea.

In view of the unsatisfactory results of corneal transplantations following corneal burns reported by Owens and others (1948) and, above all, by Stansbury (1950), a treatment which aims primarily at a maximum clarification of the affected cornea by means of conservative procedures continues to be of paramount importance. Perhaps a lamellar corneal grafting as indicated by Paufique and Philps (1950) will have a more favourable effect. Considering the findings of various colleagues and the results of new experiments on rabbits communicated by van Beek at the September meeting of the Nederlands Oogheelkundig Gezelschap at Rotterdam, and in spite of the still very doubtful theoretical foundation, treatment with Corvasymton would appear to constitute an important asset in counteracting the extremely serious effects of the severe trauma resulting from chemical burns.

Though its pharmacological action is entirely different from that of hyperaemia-inducing priscol, recommended by Nagy (1951), Corvasymton has such a convincing effect, particularly in so far as the rapid disappearance of the chemosis and the clarification of the completely opaque corneal 
regions are concerned, that, even at this stage, one is justified in giving the following broad instructions for the treatment of chemical burns:

(1) Washing with water or a neutral buffer solution, if necessary a mechanical cleansing under anaesthesia.

(2) Loosening-up of apparently necrotic conjunctival tissues, without subsequently suturing them.

(3) Plentiful instillations for a prolonged period of Corvasymton, combined with oil of vitamin $\mathrm{A}$ and mydriatics.

(4) In severe cases of corneal turbidity, paracentesis of the cornea, followed, if necessary, by irrigation of the anterior chamber of the eye, as recommended by Oaks (1945).

\section{Summary}

A case of extremely severe corrosion of both eyes by unslaked lime, treated with Corvasymton (oxyphenyl-methylamino-ethanol tartrate solution of Philips-Roxane, Limited) is reported. The visual acuity, at first limited to the perception of light in the right eye and to $2-3 / 300$ in the left eye, could be improved by contact lenses to $5 / 6.6$ and $5 / 10$ after 6 weeks of treatment, and to $5 / 10$ by ordinary spectacles after 3 months. It is argued that by loosening up the apparently necrotic conjunctival tissues and leaving them in situ, a more satisfactory result may be obtained than by transplantations of oral mucosa (Denig-Thies operation).

\section{REFERENCES}

DENIG, R. (1912). Münch. med. Wschr., 579.

Fischer, F. P., Hodgson, L., and WagenaAR, J. W. (1950). Ophthalmologica, Basel, 119, 257. Heinsius, E. (1949). Klin. Mbl. Augenheilk., 115, 673.

Hughes, W. F. (1946). Arch. Ophthal., Chicago, 35, 423.

Middleton, A. B. (1935). Illinois med. J., 67, 357.

NAGY, F. (1951). Ophthalmologica, Basel, 121, 345

OAKS, L. W. (1945). Amer. J. Ophthal., 28, 370.

Owens, W. C., Frank, J. J., Leahey, B., Messier, P. B., Scheie, H. G., Vincent, B. R., Wadsworth, J. A. C., and Stansbury, F. C. (1948). Ihid., 31, 1394.

Passow, A. (1939). Klin. Mbl. Augenheilk., 102, 431.

PAufiQue, L., and Philps, S. (1950). British Journal of Ophthalmology, 34, 746.

Pichler, A. (1910). Z. Augenheilk., 23, 397.

Stansbury, F. C. (1950). Arch. Ophthal., Chicago, 43, 337.

Thies, O. (1928). v. Graefes Arch. Ophthal., 120, 689. 